\title{
Les réformes du système éducatif allemand
}

Cécile de Bouttemont et Bernadette Plumelle

\section{(2) OpenEdition}

Journals

Édition électronique

URL : https://journals.openedition.org/ries/1802

DOI : 10.4000/ries. 1802

ISSN : 2261-4265

\section{Éditeur}

France Education international

\section{Édition imprimée}

Date de publication : 1 décembre 2002

Pagination : 12-14

ISBN : 978-2854-2-0555-8

ISSN : 1254-4590

\section{Référence électronique}

Cécile de Bouttemont et Bernadette Plumelle, "Les réformes du système éducatif allemand », Revue internationale d'éducation de Sèvres [En ligne], 31 | décembre 2002, mis en ligne le 24 novembre 2011, consulté le 06 juillet 2021. URL : http://journals.openedition.org/ries/1802 ; DOI : https://doi.org/ $10.4000 /$ ries. 1802

Ce document a été généré automatiquement le 6 juillet 2021.

(ㄷ) Tous droits réservés 


\title{
Les réformes du système éducatif allemand
}

\author{
Cécile de Bouttemont et Bernadette Plumelle
}

1 Après l'électrochoc causé par les résultats de l'étude PISA $^{1}$ sur l'évaluation des systèmes éducatifs, le gouvernement allemand met la réforme du système scolaire au cœur de ses préoccupations. Placée à la $21^{\mathrm{e}}$ position sur 32 pays, l'Allemagne se situe très largement en dessous des moyennes scolaires des pays développés en dépit de son envergure politique et économique. En outre, l'étude PISA permet de mettre en lumière des faiblesses structurelles du système éducatif, l'inadaptation de l'organisation du temps scolaire et le rapport très étroit entre réussite scolaire et origine sociale.

Dans une déclaration gouvernementale sur la "politique de l'éducation et de l'innovation ", prononcée le 13 juin 2002 devant le Bundestag, le chancelier Gerhard Schröder, se référant à l'étude PISA, remet en question l'organisation du travail scolaire qui arrête la journée de cours traditionnellement à $13 \mathrm{~h}$. Les lacunes révélées par l'enquête montrent la nécessité de consacrer plus de temps à l'enseignement des matières de base. Dans cette optique, le chancelier Gerhard Schröder propose la mise en place d'un projet visant à assurer, à partir de la rentrée 2002, des cours toute la journée dans 10000 écoles².

3 Outre l'augmentation - pour la quatrième fois consécutive - des investissements dans le domaine de l'éducation et de la recherche, la ministre fédérale, Madame Edelgard Bulmahn, envisage aussi la mise en place de trois mesures majeures ${ }^{3}$ : la redéfinition de la mission d'éducation des maternelles et garderies, qui doivent avoir pour vocation de stimuler les capacités d'apprentissage et l'esprit de curiosité des enfants; la réévaluation et l'amélioration de la formation initiale et continue des enseignants, notamment en didactique et méthodologie et, enfin, une plus grande autonomie des écoles. Au-delà de ces réformes, l'Allemagne cherche à adopter les recettes des pays qui arrivent en tête de l'enquête PISA, telles que l'articulation entre une centralisation des programmes et une plus grande indépendance des écoles ainsi qu'un apprentissage plus précoce des bases scolaires. 
4 Si les réformes sont à l'ordre du jour, leur mise en œuvre n'en reste pas moins difficile dans un pays fermement attaché au fédéralisme. D'après la Constitution, chacun des seize Länder possède l'autorité législative en matière d'enseignement; choix des programmes, préparation des examens, formation des professeurs sont des prérogatives régionales. Afin de coordonner leurs travaux, les ministres de l'Éducation des différents Länder ont institué une conférence permanente ${ }^{4}$ dont les résolutions ne peuvent être prises qu'à l'unanimité. La ministre de l'Éducation fédérale propose dans ce cadre des changements majeurs jusque-là refusés par les Länder, notamment la création d'un programme national minimum en allemand, en mathématiques et en sciences pour le secondaire (Gymnasium, Mittelschule, Realschule).

En juin 2002, la publication des résultats d'une seconde étude ${ }^{5}$ PISA, réalisée sur le seul territoire allemand et comparant les Länder entre eux, fait prendre un tour beaucoup plus politique aux réformes entreprises. Dans cette étude, les trois Länder de droite arrivent en tête du classement dans quasiment toutes les matières, ce qui, à leurs yeux, révèle l'échec d'une politique égalitariste menée par le parti social-démocrate (SPD) ${ }^{6}$. Ceux-ci s'en défendent et montrent que les Länder de gauche sont les moins riches et donc les plus prédisposés à l'échec scolaire. L'éducation devient un sujet de débat incontournable pour les différents partis (CDU, SPD, Verts). En réalité, les différences de conception de l'enseignement entre la droite et la gauche se sont largement estompées depuis les années soixante-dix au profit d'une querelle désormais axée sur le maintien d'un fédéralisme fort, défendu pas la CDU-CSU, ou d'un centralisme, prôné par le SPD. Ainsi, les solutions préconisées par le chancelier Gerhard Schröder sontelles essentiellement centralisatrices; elles portent sur la création d'un conseil pédagogique national, la rédaction de rapports annuels sur la formation et la mise en place de cadres communs au niveau national pour les programmes. La durée de scolarité obligatoire, les matières imposées et la manière d'enseigner seraient également déterminées par l'État fédéral et une institution fédérale d'évaluation serait créée pour contrôler le respect de ces minima dans chaque Land. Enfin, des tests nationaux réguliers seraient destinés à comparer les performances des élèves et des écoles.

6 En dépit des réticences des Länder quant à leur application, les «standards » minimaux au niveau national semblent acceptés. Fort de ce consensus apparent, la ministre fédérale de l'Éducation est prête à aller plus loin encore dans la centralisation avec l'uniformisation des programmes. Si aucun accord n'est trouvé entre les Länder et le Bundestag, l'administration fédérale n'exclut pas de susciter une réforme de la loi fondamentale pour donner plus de pouvoir à celui-ci en matière éducative ${ }^{7}$.

7 L'étude PISA a contribué, par son impact, à la prise de conscience des failles du système scolaire allemand mais celles-ci ne sont pas nouvelles et la nécessité d'une réforme s'imposait déjà depuis plusieurs années. Des études internationales comme IRLS (International Reading Literary Study) en 1990, et l'étude TIMSS (Third international mathematics and science study) en 1997 avaient déjà révélé les lacunes du système éducatif allemand. Réélu le 22 septembre à une courte majorité, le chancelier Schröder a pour objectif annoncé de " placer l'Allemagne dans les dix premiers pays du monde dans les dix ans». Il risque pourtant de se heurter à trois difficultés majeures: l'attachement au fédéralisme, la nécessité d'une très forte augmentation du budget de l'éducation et le recrutement des professeurs qui va se révéler crucial dans les dix années à venir. Ce cas de figure est commun à beaucoup de pays européens mais le 
corps enseignant en Allemagne est nettement plus âgé que dans d'autres pays de l'OCDE et le besoin de professeurs sera encore accru par l'ouverture des écoles toute la journée.

\section{NOTES}

1. PISA (Programm for international students assessment), publié fin 2001 par l'OCDE. On trouvera une description de l'étude PISA dans le numéro 30 de la Revue internationale d'éducation - Sèvres (p. 12-14). Par ailleurs, le site de l'OCDE propose un accès aux résultats http://www.pisa.oecd.org/

2. 4 milliards d'euros seront consacrés au soutien financier de ces écoles entre 2003 et 2007.

3. L'Observateur, $\mathrm{n}^{\circ} 231 / 232$, mai 2002.

4. KMK Kultusministerkonferenz.

5. La comparaison porte sur trois matières : allemand, mathématiques et sciences, et ceci à deux niveaux scolaires.

6. LE SPD (parti social-démocrate) s'oppose au CDU-CSU (Union chrétienne démocrate - Union chrétienne sociale) situé à droite de l'échiquier politique.

7. Jean-Luc Eluard, «Les médiocres résultats de l'enquête PISA au cœur de la campagne électorale allemande ", Agence Éducation Formation (AEF), 26/08/2002.

INDEX

Mots-clés : réforme de l'enseignement, système éducatif

Index géographique : Allemagne

\section{AUTEURS}

\section{CÉCILE DE BOUTTEMONT}

Documentaliste au centre de ressources du CIEP

BERNADETTE PLUMELLE

Responsable du centre de ressources du CIEP 